\title{
On Consistency of Decision Goals and Separability of Preferences of Decision Alternatives
}

\author{
Rudolf Felix \\ FLS Fuzzy Logik Systeme GmbH \\ Joseph-von-Fraunhofer Straße 20, 44227 Dortmund, Germany, \\ Tel. +49 231 9700921, Fax. +49 231 9700929, e-mail: felix@ fuzzy.de
}

\begin{abstract}
The paper shows how a specific notion of consistency based on a definition of interactions of decision goals is linked with the notion of separability. Subsets of decision alternatives for which additive aggregation as decision making concept is allowed are systematically identified. These subsets contain alternatives compatible with their preferences and are explicitly calculated in polynomial time. Linear preference information given separately for every single goal turns out to be sufficient in order to describe the input preferences. Despite of this linearity heuristics serving for the resolution of multidimensional decision and optimization problems with interacting goals can be formulated.
\end{abstract}

Keywords: Decision making, preference relations, separability of preferences, interactions between goals, efficient optimization heuristics.

\section{Introduction}

In decision making and optimization in many cases weighted sums are used [1], [5], [13], [18], [19], [22], [23], [24], [25]. In all such cases implicitly additive separability is thus assumed. In some other cases the separability is explicitly assumed and the approaches then explicitly deal with separable problems only, for example recently in [21]. In [2] and [3] the negative implications of this assumption is mentioned and in [6] described in detail. Basically, if there are (for instance complementary) interactions between the criteria or goals then the preferences are not additive. In some cases this fact is compensated by assuming the preference relation as given and explicitly known on all possible subsets of the set of the decision alternatives, i.e. on its power set [15]. Even if there are some interesting properties like the so-called commensurability [16], there is no escape from the combinatorial complexity of such representations of preference information. Nor is this the case if tree oriented representations of the preferences are chosen [20].

In real world decision and optimization problems however, not only the complexity from the point of view of the representation of the preferences is a problem, but also providing the preferences as input as such. This is because the stakeholders of the processes to be optimized usually are not able to provide such complex (even if it is not combinatorial) preference information in advance. Either the preference information is simply not known or it changes continuously or both. However, what can usually be provided is a preference of decision alternatives, linearly for each decision goal, decision goal by decision goal. To a certain extent this is obvious because in almost all business processes there are some so-called key performance indicators (KPIs) that are linearly expressing the positive or negative quality of possible decision options KPI by KPI. If such rankings are considered as linear preferences then the challenge is to take this minimum complex input and calculate maximum complex (optimization) decisions as if they had been calculated based on nonlinear preference information.

The paper shows how a specific decision model together with the notion of consistency based on a definition of interactions of decision goals is linked with the notion of separability. Subsets of decision alternatives for which additive aggregation as decision making concept is allowed are systematically identified. These subsets contain alternatives compatible with their preferences and are explicitly calculated in polynomial time.

Linear preference information given goal by goal for every single goal turns out to be sufficient as input. Of course, in general such kind of single goal linear preference information may be partly inconsistent with respect to the other goals. If this is the case then the inconsistencies are revealed based on the concept of interactions between the decision goals the linear preferences are given for.

Using the described decision model several complex real world optimization problems have been heuristically solved [9], [12] with efficient (polynomial) computational time [10]. The link to the notion of separability helps to understand how to formulate such heuristics.

The subsequent sections of the paper are organized as follows. First in order to provide for consistent readability of the paper a description of the concept of decision making based on interactions between goals already introduced in [7], [8] is repeated (Section 2). Then it is also repeated [11] how the concept is used in order to transform preference information into decision goals (Section 3) and how to express consistency of preferences in terms of interactions between decision goals. In Section 4 the link between the r-concistency and the separability condition is shown. In Section 5 the consequences of the results of the previous sections are discussed. Some conclusions close the paper. 


\section{Decision Making based on Interactions between Goals}

In the following four subsections the steps of decision making based on interaction between decision goals (DMIG) are described. First the notion of positive and negative impact sets is introduced. Then different types of interaction between goals are defined. After this it is shown how interactions between goals are used in order to aggregate pairs of goals to the so called local decision sets. Then it is described how the local decision sets are used for the calculation of a final decision set.

\subsection{Positive and Negative Impact Sets (Step 1)}

Before we define interactions between goals as fuzzy relations, we introduce the notion of the positive impact set and the negative impact set of a goal. A more detailed discussion can be found in [7] and [8].

Definition 1)

Definition 1a)

1. Let A be a non-empty and finite set of decision alternatives, $\mathrm{G}$ a non-empty and finite set of goals, $\mathrm{A} \cap \mathrm{G}=\varnothing, a \in A, g \in G, \delta \in(0,1]$.

For each goal $\mathrm{g}$ we define the two fuzzy sets $\mathrm{S}_{\mathrm{g}}$ and $\mathrm{D}_{\mathrm{g}}$, each from $\mathrm{A}$ into $[0,1]$ by:

Positive impact function of the goal $g: S_{g}(a):=\delta$, if a affects $g$ positively with degree $\delta$, $\mathrm{S}_{\mathrm{g}}(\mathrm{a}):=0$ else.

2. Negative impact function of the goal $g: D_{g}(a):=\delta$, if a affects $g$ negatively with degree $\delta, D_{g}(a):=0$ else.

Definition 1b)

Let $S_{g}$ and $D_{g}$ be defined as in Definition 1a). $S_{g}$ is called the positive impact set of $g$ and $D_{g}$ the negative impact set of $\mathrm{g}$.

The set $S_{g}$ contains alternatives with a positive impact on the goal $\mathrm{g}$ and $\delta$ is the degree of the positive impact. The set $D_{g}$ contains alternatives with a negative impact on the goal $g$ and $\delta$ is the degree of the negative impact.

\subsection{Interactions between Goals (Step 2)}

Let $P(A)$ be the set of all fuzzy subsets of $A$. Let $X, Y \in P(A), x$ and $y$ the membership functions of $X$ and $\mathrm{Y}$ respectively. Assume now that we have a binary fuzzy inclusion $I: P(A) \times P(A) \rightarrow[0,1]$ and a fuzzy non-inclusion $N: P(A) \times P(A) \rightarrow[0,1]$, such that $N(X, Y)=1-P(X, Y)$.

In such a case the degree of inclusions and noninclusions between the impact sets of two goals indicate the degree of the existence of interaction between these two goals. The higher the degree of inclusion between the positive impact sets of two goals, the more cooperative the interaction between them. The higher the degree of inclusion between the positive impact set of one goal and the negative impact set of the second, the more competitive the interaction. The non-inclusions are evaluated in a similar way. The higher the degree of non-inclusion between the positive impact sets of two goals the less cooperative the interaction between them. The higher the degree of non-inclusion between the positive impact set of one goal and the negative impact set of the second, the less competitive the relationship. The pair $(\mathrm{Sg}, \mathrm{Dg})$ represents the whole known impact of alternatives on the goal $\mathrm{g}$. Then $\mathrm{Sg}$ is the fuzzy set of alternatives which satisfy the goal g. Dg is the fuzzy set of alternatives, which are rather not recommendable from the point of view of satisfying the goal $\mathrm{g}$.

Based on the inclusion and non-inclusion between the impact sets of the goals as described above, 8 basic fuzzy types of interaction between goals are defined. The different types of interaction describe the spectrum from a high confluence between goals (analogy) to a strict competition (trade-off) [7].

\section{Definition 2)}

Let $\mathrm{S}_{\mathrm{g}_{1}}, \mathrm{D}_{\mathrm{g}_{1}}, \mathrm{~S}_{\mathrm{g}_{2}}$ and $\mathrm{D}_{\mathrm{g}_{2}}$ be fuzzy sets given by the corresponding membership functions as defined in Definition 1).

For simplicity we write $\mathrm{S}_{1}$ instead of $\mathrm{S}_{\mathrm{g}_{1}}$ etc.. Let

$g_{1}, g_{2}$ be elements of $G$ where $G$ is a set of goals. $T$ is a t-norm.

The fuzzy types of interaction between two goals are defined as relations which are fuzzy subsets of as follows:

$g_{1}$ is independet of $g_{2}$

$: \Leftrightarrow T\left(N\left(S_{1}, S_{2}\right), N\left(S_{1}, D_{2}\right), N\left(S_{2}, D_{1}\right), N\left(D_{1}, D_{2}\right)\right)$

$g_{1}$ assists $g_{2}: \Leftrightarrow T\left(\mathrm{I}\left(S_{1}, S_{2}\right), N\left(S_{1}, D_{2}\right)\right)$

$g_{1}$ cooperates $g_{2}: \Leftrightarrow T\left(\mathrm{I}\left(S_{1}, S_{2}\right), N\left(S_{1}, D_{2}\right), N\left(S_{2}, D_{1}\right)\right)$

$g_{1}$ is analogous to $g_{2}$

$: \Leftrightarrow T\left(\mathrm{I}\left(S_{1}, S_{2}\right), N\left(S_{1}, D_{2}\right), N\left(S_{2}, D_{1}\right), I\left(D_{1}, D_{2}\right)\right)$

$g_{1}$ hinders $g_{2}: \Leftrightarrow T\left(\mathrm{~N}\left(S_{1}, S_{2}\right), \mathrm{I}\left(S_{1}, D_{2}\right)\right)$

$g_{1}$ competes with $g_{2}$

$: \Leftrightarrow T\left(\mathrm{~N}\left(S_{1}, S_{2}\right), \mathrm{I}\left(S_{1}, D_{2}\right), \mathrm{I}\left(S_{2}, D_{1}\right)\right)$

$g_{1}$ is in trade - off to $g_{2}$

$: \Leftrightarrow T\left(\mathrm{~N}\left(S_{1}, S_{2}\right), \mathrm{I}\left(S_{1}, D_{2}\right), \mathrm{I}\left(S_{2}, D_{1}\right), \mathrm{N}\left(D_{1}, D_{2}\right)\right)$

$g_{1}$ is unspecified dependent from to $g_{2}$

$: \Leftrightarrow T\left(\mathrm{I}\left(S_{1}, S_{2}\right), \mathrm{I}\left(S_{1}, D_{2}\right), I\left(S_{2}, D_{1}\right), I\left(D_{1}, D_{2}\right)\right)$

Note that the complexity of the calculation of every type of interaction between two goals is $\mathrm{O}(\operatorname{card}(\mathrm{A}) *$ $\operatorname{card}(\mathrm{A}))=\mathrm{O}\left((\operatorname{card}(\mathrm{A}))^{2}\right)[4]$.

\subsection{Two Goals Decisions based on the Type of their Interaction and Goal Priorities (Step 3)}

The interactions between goals are crucial for an adequate orientation during the decision making process because they reflect the way the goals depend on each other and describe the pros and cons of the decision al- 
ternatives with respect to the goals. For example, for cooperative goals a conjunctive decision modeling is appropriate. If the goals are rather competitive, then decisions calculated based on an exclusive disjunction are appropriate. For a more detailed formal discussion see for instance [7], [8].

Especially in case of competitive goals for which the decision is rather an "either or" one it is needed to know which of the goals is more important than the other in order to resolve the contradiction implied by the competition. In strongly competitive decision situations this information is expressed in terms of goal priorities and then combined with the knowledge of the competition as follows: if ( $g_{1}$ is in trade-off to $\left.g_{2}\right)$ and $\left(g_{1}\right.$ is slightly more important than $\mathrm{g}_{2}$ ) then

$\mathrm{L}_{1,2}:=\mathrm{S}_{1} / \mathrm{D}_{2}$, where $\mathrm{L}_{1,2}$ is the so called local or two-goals decision set for the goals $\mathrm{g}_{1}$ and $\mathrm{g}_{2}$.

In case of very similar goals (analogous or cooperative goals) the priority information even is not necessary and the local decision set is calculated as if $\left(\mathrm{g}_{1}\right.$ cooperates with $\mathrm{g}_{2}$ ) then $\mathrm{L}_{1,2}:=\mathrm{S}_{1} \cap \mathrm{S}_{2}$ because $\mathrm{S}_{1} \cap \mathrm{S}_{2}$ surely satisfies both goals.

In this way for every pair of goals $g_{i}$ and $g_{j}$, $\mathrm{i}, \mathrm{j} \epsilon\{1, \ldots, \mathrm{n}\}$, decision sets are aggregated. The importance of goals is expressed by the so called goal priorities. A priority of a goal $\mathrm{g}_{\mathrm{i}}$ is modelled as a real number $\mathrm{P}_{\mathrm{i}} \in[0,1]$. The comparison of the priorities is modelled based on the linear ordering of the real interval $[0,1]$. The statements like $g_{i}$ is slightly more important than $\mathrm{g}_{\mathrm{j}}$ are defined as linguistic labels that simply express the extent of the difference between $P_{i}$ and $P_{j}$ (for more details see [2]).

\subsection{Final Multigoal Decision based on the Local Decision Sets (Step 4)}

The next step of the decision process is the calculation of the final multigoal decision. The calculation of the final decision is performed based on an intersection procedure of all local decision sets $\mathrm{L}_{\mathrm{i}, \mathrm{j}}$. Again a priority information assumed as given is used to build a semilinear order of the local decision sets by sorting them. The sorting process sorts the local decision sets with respect to the priorities of the goals. Subsequently an intersection set of all local decision sets is built. If this intersection set is empty then the intersection of all local decision sets except the last one in the hierarchy is built. If the resulting intersection set again is empty then the second last local decision set is excluded from the intersection process. The process iterates until the intersection is not empty (or more generally speaking until its fuzzy cardinality is big enough with respect to a given threshold). The first nonempty intersection in the iteration process is the final (multigoal) decision set and the membership values of this set give a ranking of the decision alternatives that is the result of the decision process. The set of indices $\left\{i_{1}, \ldots, i_{k}\right\}$ indicates, which set of goals $\left\{\mathrm{g}_{\mathrm{i}_{1}}, \ldots, \mathrm{g}_{\mathrm{i}_{\mathrm{k}}}\right\}$ of which local decision sets $\mathrm{L}_{\mathrm{r}, \mathrm{s}}$, $\mathrm{r}, \mathrm{s} \in\left\{\mathrm{i}_{1}, \ldots, \mathrm{i}_{\mathrm{k}}\right\}$ have contributed to $\mathrm{F}_{\{\mathrm{i} 1, \ldots, \mathrm{ik}\}}$ (for more details see [7]). Please note, that as already discussed for instance in [12] the complexity of the decision process is $\mathrm{O}\left((\operatorname{card}(\mathrm{A}))^{2} *(\operatorname{card}(\mathrm{G}))^{2}\right)$ and the complexity of the information required for the description of both the positive and the negative impact functions is $\mathrm{O}(\operatorname{card}(\mathrm{A})$ * $\operatorname{card}(\mathrm{G})$ ). Please note also that if there is no priority information given, the local decision sets $L_{i, j}$ may be sorted w.l.o.g only according to their fuzzy cardinalities.

\section{Application of the Decision Making based on In- teractions between Goals to Alternatives ranked by Reduced Preference Relations}

In preference-based decision making the input preference information has to be defined upon the power set of the decision alternatives [7]. This means that the complexity of the input information required is exponential with respect to the cardinality of the set of decision alternatives. This also means that the input information required is very difficult to obtain. Especially if the number of decision goals increases and the goals are partly conflicting the required preference information has to be multidimensional and the provider of the preference information has to express all the multidimensional interactions between the goals through the preference relation. With an increasing number of goals and interactions between them the complexity of the required input preference relation possesses the same complexity as the decision problem itself. But, if the complexity of the required input is the same as the solution of the underlying decision problem itself then the subsequent processing of the input does not really help to solve the problem and is rather obsolete.

Therefore we propose to reduce the complexity of the input preference relation. Instead of requiring a preference relation defined upon the power set of the set of the decision alternatives, which expresses the multidimensionality of the impacts of the decision alternatives on the goals, for every single decision goal a linear preference ranking of the decision alternatives with respect to that goal is required. This means that for every goal a preference ranking defined on the set of decision alternatives is required instead of a ranking defined on the power set of the alternatives. The multidimensionality of the goals is then computed from all the single goal preference rankings using the concept of interactions between the goals as defined in section 2.2.

Subsequently we extend Definition 1 . The extension defines how a single goal preference ranking defined on the set of the decision alternatives is transformed into positive and negative impact sets:

\section{Definition 1c)}

Let $\mathrm{A}$ be a non-empty and finite set of decision alternatives, $\mathrm{G}$ a non-empty, finite set of goals as defined in Definition 1a), $A \cap G=\emptyset, a \in A, g \in, \delta \in(0,1]$ Let $>_{p_{g}}$ be a preference ranking defined upon A with respect to $\mathrm{g}$ defining a total order upon $\mathrm{A}$ with respect to $\mathrm{g}$, such that $a_{i 1}>_{p_{g}} a_{i 2}>_{p_{g}} a_{i 3}>_{p_{g}} \ldots>_{p_{g}} a_{i m}$, where $\mathrm{m}=\operatorname{card}(\mathrm{A})$ and $\forall \mathrm{a}_{\mathrm{ij}}, \mathrm{a}_{\mathrm{ik}} \in \mathrm{A}, \mathrm{a}_{\mathrm{ij}}>\mathrm{p}_{\mathrm{g}} \mathrm{a}_{\mathrm{ik}}: \Leftrightarrow \mathrm{a}_{\mathrm{ij}}$ is preferred to $a_{i k}$ with respect to the goal $\mathrm{g}$. The preference relation $>_{p_{g}}$ is called the reduced single goal preference relation of the goal $\mathrm{g}$. 
For simplicity, instead of $>_{p_{g}}$ we also equivalently write RSPR of the goal $g$. All the RSPRs for all $g \in G$ are called the reduced preference relation RPR for the whole set of goals G. In order to avoid complete redundancy within the RPR we additionally define that the RSPRs of all the goals are different.

Let us assume that there is a decision situation with $n$ decision goals where $n=\operatorname{card}(G)$ and $m$ decision alternatives where $m=\operatorname{card}(\mathrm{A})$. In the subsequent we propose an additional extension of the original definition Def. $1 \mathrm{~b}$ with the aim to transform the single goal preference relations RSPR of every goals $g \in G$ into the positive and negative impact sets $\mathrm{Sg}$ and $\mathrm{Dg}$ :

\section{Defintion 1d)}

Let again A be a non-empty and finite set of decision alternatives, $\mathrm{G}$ a non-empty, finite set of goals as defined in Def. 1) a), $\mathrm{A} \cap \mathrm{B} \neq \varnothing, \mathrm{a}_{\mathrm{i}} \in \mathrm{A}, \mathrm{m}=\operatorname{card}(\mathrm{A})$, $\mathrm{g} \in \mathrm{G}, \mathrm{i}, \mathrm{c} \in\{1, \ldots, \mathrm{m}\}$. For any goal $\mathrm{g}$ we obtain both the positive and the negative impact sets $\mathrm{Sg}$ and $\mathrm{Dg}$ by defining the values of $\delta$ according to Def. 1a) and 1b) as follows:

1. For the positive impact set:

$$
\begin{gathered}
\mathrm{S}_{\mathrm{g}}\left(\mathrm{a}_{\mathrm{i}}\right)=\delta:=1 / \mathrm{i} \text { iff } \mathrm{i} \in[1, \mathrm{c}-1], \mathrm{S}_{\mathrm{g}}\left(\mathrm{a}_{\mathrm{i}}\right)=\delta:=0 \quad \text { iff } \\
\mathrm{i} \in[\mathrm{c}, \mathrm{m}] .
\end{gathered}
$$

2. For the negative impact set:

$$
\begin{gathered}
\operatorname{Dg}\left(a_{i}\right)=\delta:=0 \text { iff } i \in[1, c-1], \operatorname{Sg}\left(a_{i}\right)=\delta:=1 /(m-i+1) \text { iff } \\
i \in[c, m] .
\end{gathered}
$$

Using the Definition 1a) and Definition 1b) for any goal $\mathrm{g} \in \mathrm{G}$ we obtain a transformation of the RPR into positive and negative impact sets of all the goals and can evaluate the interactions of the goals using Definition 2 that are induced by the RPR.

Compared to classical preference based decision models this transformation helps to reduce the complexity of the input preference information required without losing modeling power for complex real world problems. The advantage is that using Definition 2, the interactions between goals that are induced by the RPR expose the incompatibilities and compatibilities that may be hidden in the RPR. The exposed incompatibilities and compatibilities are explicitly used during the further calculation of the decision sets. Note that the exposition is calculated with a polynomial number of calculation steps with degree 2 . The only additional information required from the decision maker is the priority information for each goal, which has to be expressed as a weight with a value between 0 and 1 . Many real world applications show that despite the reduced input complexity there is no substantial loss of decision quality [3].

In particular this means that it is not necessary to have classical preference relations defined upon the power set of the decision alternatives in order to handle complex decision problems with both positively and negatively interacting decision goals.

Another interesting question is how the decision making based on interactions between decision goals is formally related to decision methods based on utility functions or weighted sums. In order to investigate this we introduce the notion of r-consistency of RPRs and will consider the question under which conditions the weighted sum may be appropriate from the point of view of the application of the decision making based on interactions between goals if we have an RPR as input. For this we define the following:

\section{Definition 3)}

Given a discrete and finite set A of decision alternatives and given a discrete and finite set $\mathrm{G}$ of goals. Let $r$ $\in(0,1]$. The reduced preference relation RPR is called r-consistent $: \Leftrightarrow \exists \mathrm{A}^{\prime} \subseteq \mathrm{A}$ such that $\forall\left(\mathrm{g}_{\mathrm{i}}, \mathrm{g}_{\mathrm{j}}\right) \in \mathrm{G} \times \mathrm{G}, \mathrm{i}, \mathrm{j}$ $\in\{1, \ldots, \mathrm{k}\}, \mathrm{k} \leq \mathrm{n}, \mathrm{n}=\mathrm{card}(\mathrm{G}),\left(\mathrm{g}_{\mathrm{i}}\right.$ cooperates with $\left.\mathrm{g}_{\mathrm{j}}\right)$ $\geq \mathrm{r}$.

Intuitively, the r-consistency expresses that the RPR is at least partly consistently structured and that some of the preferences to a certain extent go along which each other. This can be seen by observing the induced interaction structure of the induced goals: If there is a subset $\mathrm{A}^{\prime} \subseteq \mathrm{A}$ for which all goals cooperate then there is some consistency of the RPR. Let us now consider an important consequence of r-consistency. The notion of $r$ consistency will later be used to characterize a new condition for the separability of the RPR in context with decisions calculated based on the interaction of decision goals. Consequently this will help to recognize to which extent weighted sum based decisions may lead to an appropriate result.

\section{The Notions r-consistency and the Separability of Preferences}

In decision making and optimization in context with multiple goals it is usually assumed that the separability condition holds and therefore utility functions based on weighted sums are a valid calculation method [6]. According to [3], for commodity choice problems separability is defined as follows:

\section{Definition 4)}

Let $M$ be a subset of the commodity set $\{1, \ldots \mathrm{n}\}$ with $\mathrm{m}<\mathrm{n}$ members and let $\sim M$ be the set of commodities not in $M$. Let the consumption set $S$ be the Cartesian product $S_{M} \times S_{\sim M}$ of possible consumption bundles of the goods in $M$ and of goods in $\sim M^{(1}$. Where $x \in S$, we write $x=\left(x_{M}, x_{\sim M}\right)$, where $x_{M}$ is an $\mathrm{m}$ vector listing a quantity of each good in $M$ and $x_{\sim M}$ lists a quantity of each good in $\sim M$.

Preferences $\mathrm{R}$ are separable on $M$ if whenever it is true that if $x=\left(x_{M}, x_{\sim M}\right) R x=\left(x^{\prime}{ }_{M}, x_{\sim M}\right)$ for some $x_{\sim M}$, it must also be that $x=\left(x_{M}, x_{\sim M}^{\prime}\right) R x=\left(x_{M}^{\prime}, x_{\sim M}^{\prime}\right)$ for all $x_{\sim M}^{\prime} \in S_{\sim M}$.

Let us now adopt Definition 4) to the decision making described in the previous section in the following way:

(1 The assumption that $S$ is the Cartesian product $S_{M}$ and $S_{\sim M}$ rules out the possibility that the possible consumptions in group $M$ depend on what is consumed in $\sim M$ or vice versa. 
Definition 5)

Definition 5a)

Let $\mathrm{G}$ be the set of goals and let $\mathrm{A}$ be the set of decision alternatives both as given in Def 1a).

Definition $5 \mathrm{~b})$

Let $G^{k}:=\left\{g_{i_{1}}, \ldots, g_{i_{k}}\right\} \subseteq \mathrm{G}$ be the set of goals that contributed to $F_{\left\{i_{1}, \ldots, i_{k}\right\}}$, as described in section $2.4, \mathrm{k} \in$ $\{1, \ldots, \mathrm{n}\}, \mathrm{n}=|G|$.

We should write $F_{G^{k}}$ for $F_{\left\{i_{1}, \ldots, i_{k}\right\}}$.

Definition 5c)

We also define $F_{\sim G^{k}}:=A \backslash F_{G^{k}}$ as the complement of $F_{G^{k}}$ and have therefore by definition that the set of decision alternatives $\mathrm{A}=F_{G^{k}} \cup F_{\sim G^{k}}$.

Let us now consider the $F_{G^{k}}$ as result of the decision making procedure as described in section 2.4. Intuitively, the higher the value of $\mathrm{k}$ for a $F_{G^{k}}$ the higher the number of goals that are simultaneously positively impacted by the decision alternatives $a \in F_{G^{k}} \subset A$. Therefore a final decision set $F_{G^{k}}$ is preferred to another final decision set $F_{G^{l}}$ if more goals are persecuted simultaneously, that means if $k>l$.

Now the definition of separability as defined in Definition 5 is used in order to formally describe how some final decision sets obtained based on interactions between decision goals may be preferred to others. For this new preference relation PREF defined upon $P(A) \times P(A)$ is introduced as follows:

\section{Definition 6)}

Let $\left(F_{G^{k}}, F_{\sim G^{k}}\right)$ and $\left(F_{G^{k}}^{\prime}, F_{\sim G^{k}}\right)$ be subsets of A. Let PREF be defined upon $P(A) \times P(A)$ as $\left(F_{G^{k}}, F_{\sim G^{k}}\right) \operatorname{PREF}\left(F_{G^{\prime}}{ }^{k^{\prime}}, F_{\sim G^{k}}\right): \Leftrightarrow$ if $k>k^{\prime}$.

Intuitively PREF means that we prefer a global decision set to another one if the decision alternatives belonging to the preferred decision set have a positive impact on more goals. Please note that the index $\mathrm{i}$ in each $F_{G}{ }^{i}$, means that $\mathrm{i}$ goals are simultaneously positively impacted by the global decision set.

With the definition made above we are now able to link the decision making based on interactions between decision goals as described in section 2 and the notion of $\mathrm{r}$-consistent reduced preference relations RPR as described in section 3 with the separability of preferences (see Definition 4) [2], [3], [6] using the understanding of preferred sets of decision alternatives as defined by the preference relation PREF.

\section{Theorem 1}

Let RPR be a reduced preference relation (see Definition1)) for a set of goals $G=\left\{g_{1}, \ldots, g_{n}\right\}$ and $\left\{F_{G^{1}}, \ldots, F_{G^{l}}\right\}$ a set of all global decision sets calculated based on the decision making as described in section 2 . Let PREF be a preference relation as defined in Definition6), then it is true that:
If the reduced preference relation RPR is r-consistent for some $\mathrm{r}>0$ then for $\mathrm{k}>1$ PREF is separable on $F_{G^{k}}$.

\section{Sketch of the proof:}

According to Definition 6) of the separability condition it has to be shown that from knowing that RPR is $\mathrm{r}$ consistent it can be shown that it is true that if $\left(F_{G^{k}}, F_{\sim G^{k}}\right)$ PREF $\left(F_{G^{k^{\prime}}}, F_{\sim G^{k}}\right)$ for some $F_{\sim G^{k}}$ it must also be $\left(F_{G^{k}}, F^{\prime}{ }_{\sim G^{k^{\prime}}}\right) \operatorname{PREF}\left(F_{G^{k^{\prime}}}, F_{\sim G^{k}}^{\prime}\right)$ for all $F_{\sim G^{k}}$.

Since RPR is $r$-consistent it is true by definition of $r$ consistency that all pairs of goals $\mathrm{g}_{\mathrm{i}}, \mathrm{i}=1, \ldots, \mathrm{k}$ cooperate. Therefore there exists a subset $\mathrm{A}^{\prime}$ of A such that all a $\in$ $\mathrm{A}^{\prime}$ have a positive impact on all goals $\mathrm{g}_{\mathrm{i}}, \mathrm{i}=1, \ldots, \mathrm{k}$. Therefore after the application of both the local decision strategies and (subsequently) the global decision strategy the intersection of all local decision sets $\mathrm{L}_{\mathrm{i}, \mathrm{j}}$ will consist exactly of all a $\in \mathrm{A}^{\prime}$ (with a membership $>0$ ). Therefore we have that $F_{G^{k}}$, is maximum possible for the simultaneous persecution of the $\mathrm{k}$ goals. This again means that if any other $\mathrm{A}$ would be added to $\mathrm{A}^{\prime}$ (let us call this new set $\mathrm{A}^{\prime \prime}$ ) then no additional goal of the $\mathrm{k}$ goals would be simultaneously positively impacted by the elements of $\mathrm{A}^{\prime \prime}$. This means that in this sense $\mathrm{A}^{\prime}$ is maximal because it will always be that for a $F^{\prime}{ }_{G^{\prime}}$, derived from A" compared to $F_{G^{k}}$ it will always be $\mathrm{k}^{\prime}<\mathrm{k}$. Therefore $F_{G^{k}}$ will always be preferred to any $F_{G^{k}}^{\prime}$, in the sense of the definition of PREF and consequently the expression

$\left(F_{G^{k}}, F_{\sim G^{k^{\prime}}}^{\prime}\right) \operatorname{PREF}\left({F^{\prime}}_{G^{k^{\prime}}}, F^{\prime}{ }_{\sim G^{k}}\right)$ is true for all $F_{\sim G^{k}}^{\prime}$.

\section{Discussion of the Consequences}

The notion of $r$-consistency provides a possibility to quantify the consistency within the RPR and therefore the interaction between the RSPRs that express the compatibility of the preferences over the RSPRs. The higher the number $\mathrm{k}$ of goals for which the $\mathrm{r}$ consistency condition holds the better the compatibility of the RPR and therefore of the RSPRs compared to each other. More exactly: Theorem 1 shows that $\mathrm{r}$ consistency on the RPR implies the separability of the preference relation PREF on the subset $F_{G^{k}}=\mathrm{A}^{\prime} \subseteq \mathrm{A}$. This again shows that $\mathrm{r}$-consistency helps to understand, which part of the RPR can be considered as additively separable. Since r-compatibility is defined based on the notion of cooperating decision goals Theorem 1 gives a link between the utility function or weighted sum based aggregation of preferences and the decision making based on interactions between goals (DMIG). In [11] a link to the theory of matroids [17] was discussed. With the result of this paper a relation to the notion of separability is shown.

In section 3 it is described how a reduced preference relation RPR can be obtained from single goal preference relations RSPR, even if the given decision problem may be multidimensional and the goals may partly positively and partly negatively interact instead of re- 
quiring a preference relation defined upon the power set of the set of the decision alternatives in advance. It turns out that for every single decision goal of the decision alternatives with respect to every single goal only a linear preference relation is required as apriori input. Although the input complexity required about the preferences is linear, applying DMIG provides a method that reveals how the hidden multidimensionality of the consequences of the preference structure should imply the calculation of decision [11] (in [11] it is also shown that this can be done with polynomial computation time). Using the result of Theorem 1 it is possible to identify a subset of A for which additive separable aggregation is not allowed. This is namely the difference set $\mathrm{A} \backslash \mathrm{A}^{\prime}$. Please note that in real world applications such information derived in advance is a substantial advantage because providing single goal preferences is usually possible whereas providing multidimensional preferences as input is not. Please note also that the remaining $n-k$ goals for which the $r$-consistency condition does not hold usually are conflicting. Therefore an aggregation of decisions based for these goals is not allowed. This means that if the r-consistency does not hold for k-n goals then the input preference information may contain conflicts (being partly inconsistent). These conflicts are recognized and balanced by DMIG, too. The recognition is provided by the calculation of negative interactions as also defined in section 2.2. The interaction of the type "compete" is an example, the interaction of the type "trade-off" another one. This result is similar to [14] where the separability of classes of domain competence is measured for a particular application case. However, compared to [14] the method presented here is rather domain independent.

In [12] it is described how DMIG is used for the resolution of the optimization of a complex real world resource optimization problem. In the context of such problems the goal set $\mathrm{G}$ usually can be understood as derived from key performance indicators (KPIs) that describe the quality of the business process to be optimized. Hence there is a direct correlation between $G$ and the KPIs of the process to be optimized. The input information required for such optimization until now had to be numerically prepared in the sense of fuzzy impact functions defined in the value domains of the KPIs. Instead of this, based on RPR the complexity of the input information required may now be reduced to the calculation of reduced single goal preference relations RSPR. This is easier because the decision alternatives that have to be analyzed in the optimization process require only to be ranked according to their single goal/KPI preferences without substantial loss of the quality of the optimization result. More complex interactions between the preferences are revealed and are not required in advance.

Optimization heuristic algorithms like that described in [12] iterate navigating step by step through the optimization space implied by the goals and the RPR. Because of Theorem 1 it is clear that such optimization algorithms may concentrate only on the separable subset of decision alternatives, which as such are provided by DMIG and therefore by the analysis of interaction between decision goals. This result is similar to the result presented by Carbonell [4] in the field of data mining in Bioinformatics where partial additive separability is assumed by considering only particular interrelations among gene data. These particular interrelations there are comparable with the particular type of interactions between decision goals, namely its cooperation, here.

\section{Conclusions}

The paper shows how the notion of r-consistency based on a formal definition of interactions of decision goals introduced in DMIG is related to the notion of separability of the preferences of decision sets. It turns out that r-consistency helps to identify a subset of decision alternatives for which additive aggregation is allowed. This identifies a link between preference relations, the separability condition and decision making as formalized in DMIG. Using that link subsets of decision alternatives compatible with their preferences can be explicitly calculated in polynomial time and therefore efficiently. Preference information required as input is reduced to linear rankings of the decision alternatives. More complex interactions between the preferences are revealed and are not required in advance. The link between r-consistency, separability and DMIG helps to formulate efficient optimization heuristics that adequately navigate between additively separable parts of the decision or optimization space. Many heuristics based on DMIG that could successfully be applied in numerous real world applications underline the suitability of the r-consistency condition.

\section{References}

[1] Abdel-Kader, R.: Fuzzy Particle Swarm Optimization with Simulated Annealing and Neighborhood Information Communication for Solving TSP. In: (IJACSA) International Journal of Advanced Computer Science and Applications, Vol. 2, No. 5, (2011)

[2] Bergstrom, T.: Lecture Notes on Separable Preferences. In: UCSB Econ 210A, www.econ.ucsb.edu/ tedb/Courses/GraduateTheory UCSB/separabilitynotes.pdf

[3] Bergstrom, T.: Notes on Uncertainty and Expected Utility. In: UCSB Econ 210A, (2012)

[4] Carbonell, P.: Efficient Fuzzy Logic-Based Algorithm for Microarray Network Identification and Prediction in Bioinformatics. In: Electronics and Electrical Engineering, Nr. 3 (67), 2006.

[5] Davarynejad, M. and Akbarzadeh-T, M.-R.: A Novel General Framework for Evolutionary Optimization: Adaptive Fuzzy Fitness Granulation. In: Proceedings of the 2007 Congress of Evolutionary Computation (CEC 2007), (2007)

[6] Deaton, A., Muellbauer, J.: Economics and consumer behavior, Cambridge University Press, New York, (1980)

[7] Felix, R.: Relationships between goals in multiple attribute decision making. In: Fuzzy Sets and Systems, Vol.67, 47-52, (1994) 
[8] Felix, R.: Decision-making with interacting goals.In: Handbook of Fuzzy Computation, Ruspini, E., Bonissone, P.P, Pedrycz, W. (Eds.), IOP Publishing Ltd, (1998)

[9] Felix, R.: Real World Applications of a Fuzzy Decision Model Based on Relationships between Goals (DMRG). In: Forging the New Frontiers, Fuzzy Pioneers I (1965-2005), Vol. 217, 351-367, Springer Verlag in the series Studies in Fuzziness and Soft Computing, (October 2007)

[10]Felix, R.: Multicriterial Decision Making (MCDM): Management of Aggregation Complexity Through Fuzzy Interactions Between Goals or Criteria. In: Proceedings of the 12th International IPMU Conference, Málaga, Spain, (2008)

[11]Felix, R.: Multi-Goal Aggregation of Reduced Preference Relations Based on Fuzzy Interactions between Decision Goals. In: Proceedings of the IFSA World Congress, Lisbon, Portugal, (2009)

[12]Felix, R.: Optimization of Partly Conflicting Goals in Complex Resource Planning, 8th Conference of the European Society for Fuzzy Logic and Technology (EUSFLAT 2013) Milano (2013)

[13] Krivy, I.: Optimization of fuzzy models using evolutionary algorithms. In: Proceedings of the 7th International Conference APLIMAT, (2008)

[14]Luengo, J. and Herrera, F.: On the use of Measures of Separability of Classes to characterize the Domains of Competence of a Fuzzy Rule Based Classification System. In: Proceedings of the IFSA World Congress, Lisbon, Portugal, (2009)

[15] Modave, F., Dubois, D., Grabisch, M. and Prade, H.: A Choquet integral representation in multicriteria decision making. In: AAAI Fall Symposium, Boston, Ma, November (1997)
[16] Modave, F. and Grabisch, M.: Preference representation by a Choquet integral: Commensurability hypothesis. In: Proceedings of the 7th International IPMU Conference, Paris, France, 164-171, (1998)

[17] Oxley, J.: Matroid Theory. In: Oxford University Press, (1992)

[18] Pandian, P.: Multi-objective Programming Approach for Fuzzy Linear Programming Problems. In: Applied Mathematical Sciences, Vol. 7, 2013, no. 37, 1811-1817, HIKARI Ltd

[19]Pedrycz, W.: Fuzzy Evolutionary Computation. Springer Science+Business Media, (1997)

[20] Saaty, T. L.: The Analytic Hierarchy Process. In: Mc Graw-Hill, (1980)

[21] Sen, S. and Pal, B.B.: A Piecewise Approximation Method to Solve Fuzzy Separable Quadratic Programming Problems. In: International Journal of Advanced Computer Research, Vol. 3, No.1, Issue 08.03.2013

[22] Song W., Cheon Choi L., Cheol Park S., Feng ding X.: Fuzzy evolutionary optimization modeling and its applications to unsupervised categorization and extractive summarization. In: Elsevier, Expert Systems with Applications 38 (2011)

[23] Torra, V.: Weighted OWA operators for synthesis of information. In: Proceedings of the fifth IEEE International Conference on Fuzzy Systems, New Orleans, USA, 966-971, (1996)

[24] Wang, X.-Y., Garibaldi, J.M.: Simulated Annealing Fuzzy Clustering in Cancer Diagnosis. In: Informatica 29, (2005)

[25] Yager, R.R.: Families of OWA operators. In: Fuzzy Sets and Systems, Vol. 59, 125-148, (1993) 